\title{
Dynamics of Screening in Modified Gravity
}

\author{
Lotte ter Haar@,$^{1,2}$ Miguel Bezares $\odot,{ }^{1,2}$ Marco Crisostomi $\odot,{ }^{1,2}$ Enrico Barausse $\odot^{1,2}$ and Carlos Palenzuela $\oplus^{3,4}$ \\ ${ }^{1}$ SISSA, Via Bonomea 265, 34136 Trieste, Italy and INFN Sezione di Trieste, via Valerio 2, 34127 Trieste, Italy \\ ${ }^{2}$ IFPU-Institute for Fundamental Physics of the Universe, Via Beirut 2, 34014 Trieste, Italy \\ ${ }^{3}$ Departament de Física, Universitat de les Illes Balears and Institut d'Estudis Espacials de Catalunya, \\ Palma de Mallorca, Baleares E-07122, Spain \\ ${ }^{4}$ Institut Aplicacions Computationals (IAC3), Universitat de les Illes Balears, Palma de Mallorca, Baleares E-07122, Spain
}

(Received 7 September 2020; accepted 21 January 2021; published 1 March 2021)

\begin{abstract}
Gravitational theories differing from general relativity may explain the accelerated expansion of the Universe without a cosmological constant. However, to pass local gravitational tests, a "screening mechanism" is needed to suppress, on small scales, the fifth force driving the cosmological acceleration. We consider the simplest of these theories, i.e., a scalar-tensor theory with first-order derivative selfinteractions, and study isolated (static and spherically symmetric) nonrelativistic and relativistic stars. We produce screened solutions and use them as initial data for nonlinear numerical evolutions in spherical symmetry. We find that these solutions are stable under large initial perturbations, as long as they do not cause gravitational collapse. When gravitational collapse is triggered, the characteristic speeds of the scalar evolution equation diverge, even before apparent black-hole or sound horizons form. This casts doubts on whether the dynamical evolution of screened stars may be predicted in these effective field theories.
\end{abstract}

DOI: 10.1103/PhysRevLett.126.091102

Introduction.-The accelerated expansion of the universe is among the biggest mysteries of cosmology. While achievable by a cosmological constant or a dark-energy (DE) component, these possibilities face long-standing theoretical issues [1]. Thus, the possibility that cosmic acceleration may arise from a modification of general relativity (GR) on cosmological scales has attracted considerable attention [2].

The simplest extension of GR is provided by scalartensor theories, where gravity is described not only by two tensor polarizations but also by a scalar graviton. Their most general form is given by degenerate higher-order scalar-tensor theories [3], which contain well-known examples such as the Fierz-Jordan-Brans-Dicke (FJBD) theory [4-6], the dilatonic Gauss-Bonnet theory [7], Horndeski [8] and beyond-Horndeski [9] theories, etc.

While scalar-tensor theories can produce self-accelerated cosmic expansion without a cosmological constant [10], they typically also produce local deviations from GR on small scales [11]. These include the solar system and binary pulsars, where GR has been tested to exquisite accuracy [12-14], and the compact-object binaries observed by gravitational-wave (GW) interferometers [15]. However, some theories possess "screening mechanisms" (Vainshtein screening [16,17], $k$-mouflage [18], chameleon or symmetron screening $[19,20]$, etc.) that locally produce a GR-like phenomenology, potentially passing existing constraints. Screening has only been tested in static or quasistatic configurations, but its validity is also often taken for granted in dynamical settings, e.g., GW generation [21]. Here, we will verify this assumption.
We consider scalar-tensor theories with first-order derivative self-interactions ( $k$-essence $[22,23]$ ). Among the many theories aiming to explain DE, $k$-essence is among the few unconstrained by the GW170817 bound on the GW speed [24,25] and by other constraints based on GW propagation [26-28]. By studying static and spherically symmetric solutions, we confirm the presence of "kinetic" screening ( $k$-mouflage [18]) in nonrelativistic stars, and extend it to fully relativistic, compact stars. Then, we consider spherically symmetric time evolutions of these screened solutions, using the fully nonlinear code of [29].

Static spherically symmetric screening.-With units $\hbar=c=1$ and signature $(-+++)$, the $k$-essence action in the Einstein frame is

$$
S=\int d^{4} x \sqrt{-\tilde{g}}\left[\frac{M_{\mathrm{Pl}}^{2}}{2} \tilde{R}+K(\tilde{X})\right]+S_{m}\left[g_{\mu \nu}, \Psi\right]
$$

Here, $M_{\mathrm{Pl}}=(8 \pi G)^{-1 / 2}$ is the Planck mass; $g_{\mu \nu}=A(\phi) \tilde{g}_{\mu \nu}$ and $\tilde{g}_{\mu \nu}$ are, respectively, the metrics in the Jordan and Einstein frames; the conformal factor is $A(\phi)=e^{\alpha \phi / M_{\mathrm{Pl}}}$, where $\alpha \sim \mathcal{O}(1)$ is dimensionless; $\tilde{g}$ and $\tilde{R}$ are the (Einsteinframe) metric determinant and Ricci scalar; $\tilde{X} \equiv \tilde{\nabla}_{\mu} \phi \tilde{\nabla}^{\mu} \phi$ is the standard kinetic term of the scalar field $\phi$. Variation of the action yields

$$
\tilde{G}_{\mu \nu}=8 \pi G\left[K(\tilde{X}) \tilde{g}_{\mu \nu}-2 K^{\prime}(\tilde{X}) \tilde{\nabla}_{\mu} \phi \tilde{\nabla}_{\nu} \phi+\tilde{T}_{\mu \nu}\right],
$$




$$
\tilde{\nabla}_{\mu}\left[K^{\prime}(\tilde{X}) \tilde{\nabla}^{\mu} \phi\right]=\frac{1}{4} A^{-1}(\phi) A^{\prime}(\phi) \tilde{T},
$$

where $\tilde{G}_{\mu \nu}$ and $\tilde{T}_{\mu \nu}$ are, respectively, the Einstein and energy-momentum tensors in the Einstein frame [30].

For $K(\tilde{X})$ we consider only the lowest-order terms

$$
K(\tilde{X})=-\frac{1}{2} \tilde{X}+\frac{\beta}{4 \Lambda^{4}} \tilde{X}^{2}-\frac{\gamma}{8 \Lambda^{8}} \tilde{X}^{3},
$$

with $\Lambda$ the strong-coupling scale of the effective field theory (EFT), and $\beta, \gamma \sim \mathcal{O}(1)$ dimensionless coefficients. We assume that the background scalar field is responsible for DE, therefore, $\Lambda \sim\left(H_{0} M_{\mathrm{Pl}}\right)^{1 / 2} \sim 5 \times 10^{-3} \mathrm{eV}$, where $H_{0}$ is the present-day Hubble expansion rate. It is exactly the hierarchy $M_{\mathrm{Pl}} \gg \Lambda$, needed for cosmology, that allows for screening local scales (a much larger $\Lambda$ would make $k$-essence equivalent to FJBD, where no screening appears). Screened solutions are possible for any $\beta<$ $0, \gamma>0$ [31], but in the following, we set $\beta=0$ and $\gamma=1$, which ensures that $1+2 X K^{\prime \prime}(X) / K^{\prime}(X)>0$ for all $X$ (a sufficient condition to avoid Tricomi-type breakdowns of the Cauchy problem [29]; see, also, [36,37]). Our conclusions also hold for more general $\beta$ and $\gamma$, if they are such that this condition holds (see examples in [29]).

In more detail, [18] suggested that nonrelativistic stars in $k$-essence present a $k$-mouflage mechanism, whereby GR is recovered within a "screening radius" $r_{k} \sim \Lambda^{-1} \sqrt{M / M_{\mathrm{Pl}}}$ (with $M$ the star's mass), as a result of the nonlinear terms in Eq. (4) dominating over the linear one [38]. To check this, first, we consider constant-density, nonrelativistic stars. Using the same weak-field approximation applied in $[41,42]$ to study screening in massive (bi-)gravity, we obtain an approximate equation for the scalar-field radial derivatives $y \equiv \phi^{\prime}$ and $y^{\prime}\left(\right.$ with $\left.{ }^{\prime} \equiv d / d r\right)$

$$
\begin{aligned}
\frac{r \rho}{M_{\mathrm{Pl}} \Lambda^{2}}= & y^{\prime}\left[\frac{\left(3 \alpha^{2}+2\right) r}{\alpha \Lambda^{2}}+\frac{r^{2} y}{M_{\mathrm{Pl}} \Lambda^{2}}+\frac{15 \gamma r y^{4}\left(2 M_{\mathrm{Pl}}+\alpha r y\right)}{4 M_{\mathrm{Pl}} \alpha \Lambda^{10}}\right] \\
& +\frac{2\left(3 \alpha^{2}+2\right) y}{\alpha \Lambda^{2}}+\frac{2 \alpha^{2} r y^{2}}{M_{\mathrm{Pl}} \Lambda^{2}}+\frac{3 \gamma y^{5}}{\alpha \Lambda^{10}}+\frac{3 \gamma r y^{6}}{M_{\mathrm{Pl}} \Lambda^{10}} \\
& +\frac{5 \alpha \gamma r^{2} y^{7}}{4 M_{\mathrm{Pl}}^{2} \Lambda^{10}} .
\end{aligned}
$$

Approximate analytic solutions to this equation can be obtained in the stellar interior: $y_{1} \approx\left[\alpha \rho r \Lambda^{8} /\left(3 \gamma M_{\mathrm{Pl}}\right)\right]^{1 / 5}$; in the exterior within the screening radius: $y_{2} \approx$ $\left[\alpha M \Lambda^{8} /\left(4 \pi \gamma M_{\mathrm{Pl}} r^{2}\right)\right]^{1 / 5}$; and outside the screening radius: $y_{3} \approx$ const $/ r^{2}$. In the FJBD case $\beta=\gamma=0$, an approximate solution is given by $y_{3}$ outside the star and by $y_{0} \approx$ $\alpha \rho r /\left[2 M_{\mathrm{Pl}}\left(2+3 \alpha^{2}\right)\right]$ inside.

These approximate solutions show that, in $k$-essence, the scalar derivative (which encodes the additional "fifth force" beyond GR) is suppressed inside $r_{k}$. However, the inner solution is not regular at the star's center. Regularity requires $y=\phi^{\prime} \propto r$ when $r \rightarrow 0$, and a different behavior is not acceptable, as it would cause the appearance of a central conical singularity.

To amend this behavior, we numerically solve Eq. (5), imposing $y \rightarrow 0$ when $r \rightarrow 0$ as a boundary condition. This completely determines the solution as Eq. (5) does not involve $y^{\prime \prime}$. Thus, it is not trivial that the regular solution will match the approximate ones $\left(y_{1}, y_{2}, y_{3}\right)$ above. In more detail, since Eq. (5) is singular at $r=0$, we must solve it perturbatively at small radii, imposing $y \propto r$ when $r \rightarrow 0$. This yields another approximate solution, (which at leading order, matches the approximate FJBD inner solution $y_{0}$ ) which we use to "inch away" from $r=0$ and provide initial conditions for the numerical integration. This procedure gives the numerical solution (regular at the center) shown by a solid line in Fig. 1 (left panel), where we also compare to the approximate solutions $y_{0}, y_{1}, y_{2}$, and $y_{3}$. As can be seen, the regular numerical solution matches the approximate solutions $y_{1}, y_{2}$, and $y_{3}$ everywhere but near the center, where we find agreement with $y_{0}$ (the FJBD solution), instead.

These results confirm the existence of (regular) $k$-mouflage solutions in nonrelativistic stars, but it is not obvious that the same will apply to strongly gravitating relativistic stars, e.g., neutron stars, or even for weakly gravitating stars when the full system (2)-(3) is solved simultaneously. Therefore, we write Eqs. (2) and (3) using a spherically symmetric ansatz for the (Einstein-frame) metric $d \tilde{s}^{2}=\tilde{g}_{t t}(\tilde{r}) d t^{2}+\tilde{g}_{\tilde{r} \tilde{r}}(\tilde{r}) d \tilde{r}^{2}+\tilde{r}^{2} d \Omega^{2}$ and for the scalar field and solve the coupled system by imposing regularity at the center. Since Eqs. (2) and (3) depend on $\phi$ [and not only on $\phi^{\prime}$ and $\phi^{\prime \prime}$, unlike Eq. (5)], an additional boundary condition is needed for $\phi$. Thus, we require $\phi$ to approach a constant $\phi_{\infty}$ at spatial infinity. If we take $\left|\phi_{\infty}\right| / \Lambda \lesssim 1$, as expected from cosmological considerations, results are robust against the exact value of $\phi_{\infty}$.

We adopt a polytropic equation of state $p=K \rho_{b}^{\Gamma}$, $p=(\Gamma-1)\left(\rho-\rho_{b}\right)$ - with $p, \rho, \rho_{b}$, the pressure, energy density and baryonic density - in the Jordan frame (thus, the equation of state in the Einstein frame involves the conformal factor, cf. [32,33]). We use $K=123 G^{3} M_{\odot}^{2} / c^{6}$ and $\Gamma=2$ for neutron stars, and $K=5.9 \times 10^{-3} G^{1 / 3} R_{\odot}^{2 / 3} / c^{2 / 3}$ and $\Gamma=4 / 3$ for weakly gravitating, Sun-like stars. We impose regularity by perturbatively solving the equations near the center, and use this solution to provide initial conditions for the outbound integration at small but nonzero $r$. These initial conditions depend on the central values of the scalar field and density. We fix the former via a shooting procedure by requiring $\phi \rightarrow \phi_{\infty}$ as $r \rightarrow \infty$, while the central density is varied on a grid to produce stars of different masses.

The solution for a Sun-like star is shown in Fig. 1 (left panel, dotted orange line), and presents the same qualitative features as the approximate solution obtained previously. Similarly, the radial profile of $\phi^{\prime}$ for neutron stars (right panel, solid orange line) shows kinks right outside the 

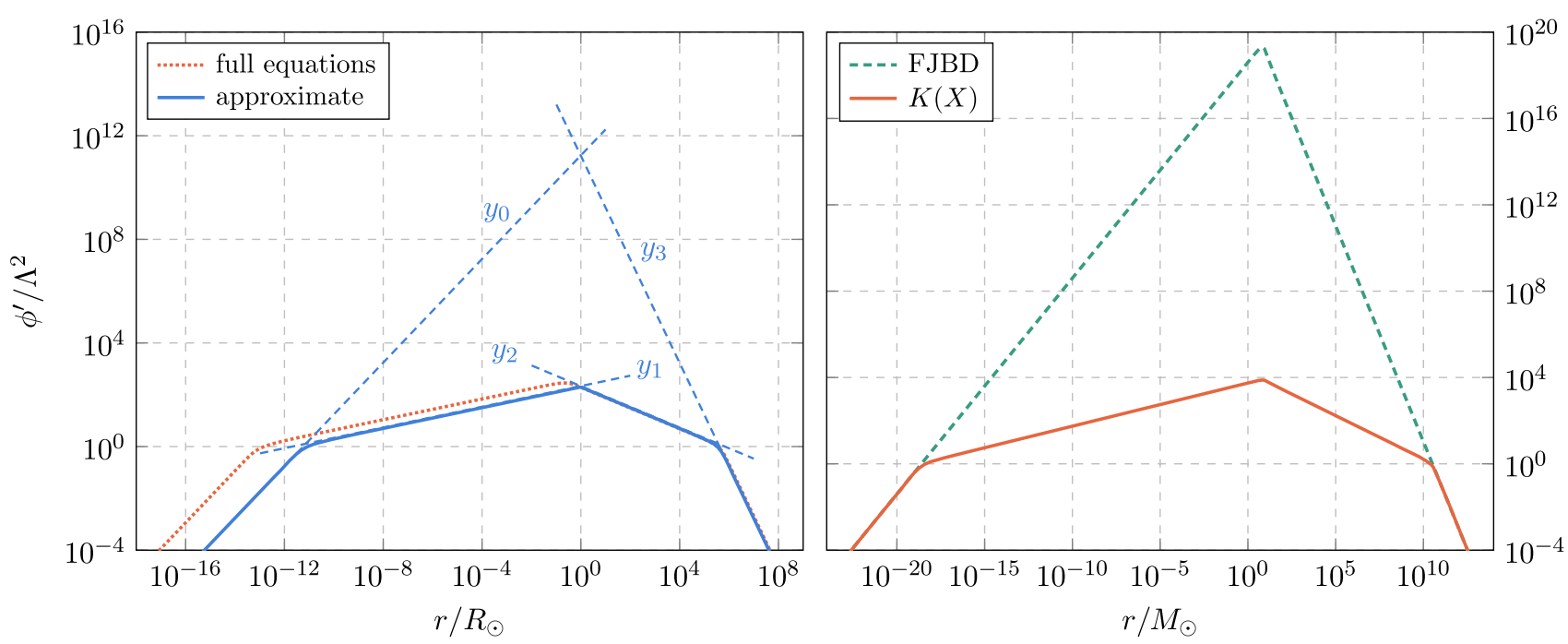

FIG. 1. $\phi^{\prime}$ vs $r$ for $\gamma=1$ and $\alpha=1 / 2$. Left: A weakly gravitating, Sun-like star. We plot the numerical solution of Eq. (5) (solid blue line), the approximate solutions $y_{0}, y_{1}, y_{2}, y_{3}$ (dashed blue lines), and the numerical solution of the full system Eqs. (2) and (3) (dotted orange line). Right: A neutron star in $k$-essence (solid orange line) and $\operatorname{FJBD}(\beta=\gamma=0$, dashed green line).

center, at the stellar surface, and at the screening radius. We also plot, by a dashed green line, the solution to Eq. (2)-(3) obtained for $\beta=\gamma=0$ (i.e., FJBD). The $k$ mouflage solution matches the FJBD one near the center and outside $r_{k}$, but deviates from it (suppressing $\phi^{\prime}$ and, thus, the scalar force) when nonlinearities become important (i.e., when $X / \Lambda^{4} \gtrsim 1$ ). Similar plots and conclusions apply to generic $\beta<0$ and $\gamma>0$.

Again, for neutron stars, in Fig. 2 (left panel), we show the ratio of the Newtonian force $|d U / d r|$, with $U=-\left(g_{t t}+1\right) / 2$ the Newtonian potential, for solutions in $k$-essence and FJBD theory with respect to solutions in $\mathrm{GR}$, as a function of the Jordan-frame areal radius $r$.
Note that the scalar-field contribution (fifth force) is suppressed in $k$-essence relative to FJBD theory inside $r_{k}$, as expected from screening. In Fig. 2 (right panel), we also show the fractional deviations of the (Jordan-frame) metric components $g_{t t}$ and $g_{r r}$ from GR, in FJBD theory (with $\alpha=1 / 2$ and $\alpha=5 \times 10^{-3}$ ) and in $k$-essence (with $\alpha=1 / 2$ ). Note that the tiny deviations from GR in $k$ essence suggest that not only is the Newtonian dynamics essentially equivalent to GR's but that the same also holds at first post-Newtonian order. This is apparent from the comparison with FJBD theory with $\alpha=5 \times 10^{-3}$, which is in agreement with current solar system tests of the postNewtonian dynamics $[13,14,32,33]$.
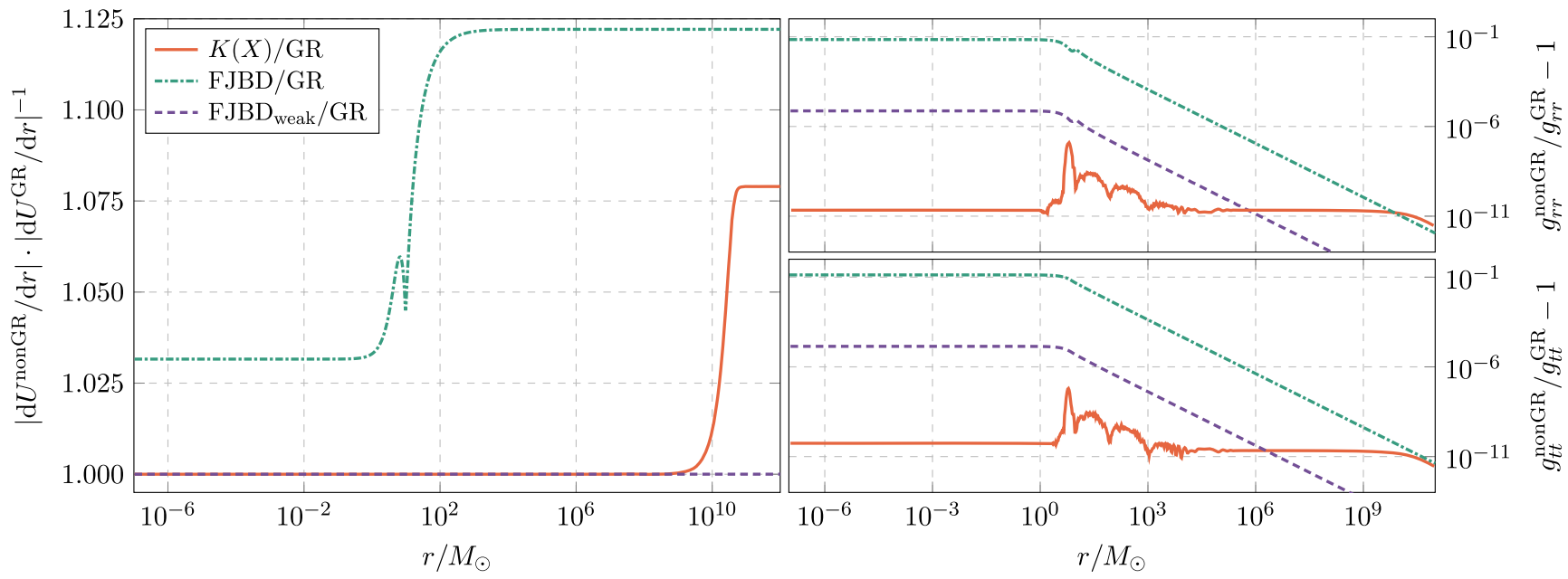

FIG. 2. Deviations of the metric and its derivatives from GR, for $k$-essence with $\gamma=1$ and $\alpha=1 / 2$, and for FJBD (with $\alpha=1 / 2$ and $\alpha=5 \times 10^{-3}$, the latter referred to as FJBD ${ }_{\text {weak }}$ ). Left: Ratio of the Jordan-frame Newtonian forces with the GR counterpart. The FJBD star is slightly smaller than in GR, which explains the feature at $r / M_{\odot} \sim 10$. Right: The fractional deviation from GR of the $g_{r r}$ (upper panel) and $g_{t t}$ (lower panel) components of the Jordan-frame metric. 
Screening perturbations and time evolutions.-To check the stability of our static spherical solutions, we numerically evolve the scalar, the metric, and the matter fields according to Eqs. (2) and (3). We employ the $1+1$ (i.e., spherically symmetric but time-dependent) fully nonlinear evolution code used in [29] for the vacuum case, supplementing it with matter as described in [43]. Both the matter's and the scalar's evolution are expressed as conservation laws and integrated with high-resolution shockcapturing (HRSC) methods. First, we checked that, if static spherical solutions (for both Sun-like and neutron stars) are used as initial data, the system does not evolve (e.g., case A in Fig. 3) [44]. However, if we perturb them (in their matter or scalar content), the results vary dramatically according to $\Lambda$ and the perturbation amplitude or sign.

For $\Lambda \gtrsim 10^{7} \mathrm{eV}$, the static spherical initial data show no screening and are very similar to FJBD theory, as expected. Nonlinearities in the scalar sector are never excited and evolutions are well behaved, however large the initial perturbations. For screened solutions $\left(\Lambda \lesssim 10^{6} \mathrm{eV}\right)$, the outcome of time evolutions depends on the initial perturbation amplitude or sign. Small perturbations (case B in Fig. 3) and large ones initially decreasing the stellar compactness (case $\mathrm{C}$ in Fig. 3) oscillate but do not grow, confirming the stability of the screened solutions. However, when large perturbations have the right sign to trigger gravitational collapse (case D in Fig. 3), the characteristic propagation speeds of the scalar-field equation eventually diverge, even before apparent or black-hole horizons form. In more detail, the characteristic speeds are encoded in the principal part (i.e., the part involving only the highest derivatives) of Eq. (3), which is given by $\gamma^{\mu \nu} \tilde{\nabla}_{\mu} \tilde{\nabla}_{\nu} \phi$, with

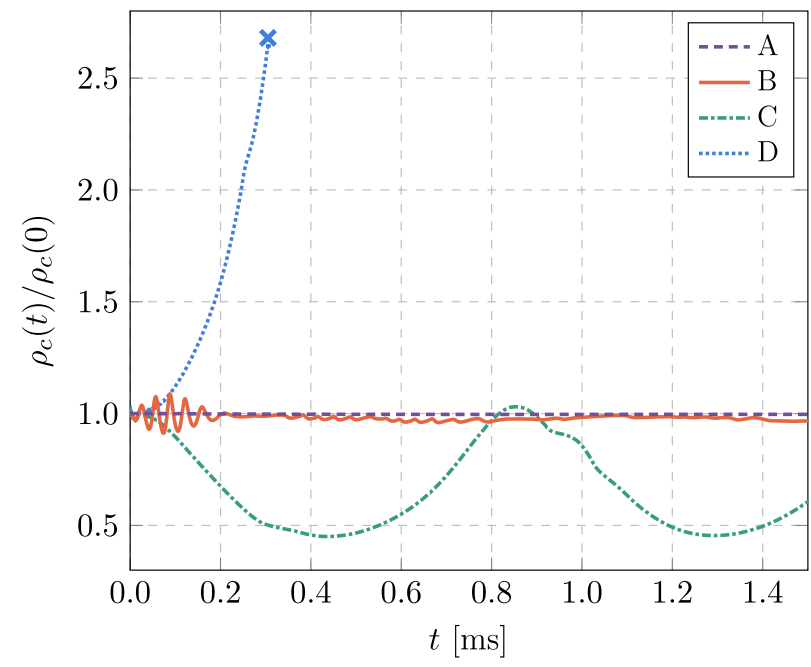

FIG. 3. Evolution of the (Einstein-frame) central density of a $k$-mouflage star for $\Lambda \simeq 10^{6} \mathrm{eV}$ (with $\alpha \simeq 0.2$ and $\gamma=1$ ), for unperturbed initial data (A); small initial perturbations (B); large perturbations that initially decrease (C) or increase (D) the star's compactness. Case D leads to collapse and diverging characteristic speeds (at the time marked by a cross). $\gamma^{\mu \nu} \equiv \tilde{g}^{\mu \nu}+2\left[K^{\prime \prime}(\tilde{X}) / K^{\prime}(\tilde{X})\right] \tilde{\nabla}^{\mu} \phi \tilde{\nabla}^{\nu} \phi$. Writing the principal part in first-order form, i.e., $\partial_{t} \boldsymbol{U}+\boldsymbol{V} \partial_{r} \boldsymbol{U}$, with $\boldsymbol{U} \equiv\left(\partial_{t} \phi, \partial_{r} \phi\right)$ and $\boldsymbol{V}$ the characteristic matrix, the characteristic speeds are then the eigenvalues of $\boldsymbol{V}$ [29]

$$
v_{ \pm}=-\frac{\gamma^{t r}}{\gamma^{t t}} \pm \sqrt{\frac{-\operatorname{det}\left(\gamma^{\mu \nu}\right)}{\left(\gamma^{t t}\right)^{2}}} .
$$

Their nonlinear divergence, appearing because $\gamma^{t t} \rightarrow 0$, is also known to plague $k$-essence in vacuum (for initial data close to critical collapse) $[29,47,48]$ and resembles that of the Keldysh equation $t \partial_{t}^{2} \phi(t, r)+\partial_{r}^{2} \phi(t, r)=0$, which is hyperbolic with characteristic speeds $\pm(-t)^{-1 / 2}$ for $t<0$. The problem persists when looking for screened solutions through relaxation of GR stars, as done in [32].

As we stressed in [29], diverging characteristic speeds are not necessarily pathological and may occur because of gauge choices (see, e.g., a wave equation on flat space in Eddington-Finkelstein coordinates, $d s^{2}=-d v^{2}+2 d v d r+$ $r^{2} d \Omega^{2}$ ). As in vacuum [29], the characteristic speeds may be kept finite during the evolution if a nonvanishing shift in the metric is allowed. Nevertheless, because of the nonlinear nature of the field equations, we could not identify a suitable coordinate condition (i.e., a choice of lapse and/or shift) avoiding these divergences and simultaneously producing stable evolutions at least in $1+1$ dimensions. We tried different shift conditions that successfully keep the velocities finite, but those still lead to unstable evolutions even with HRSC methods. Whatever its interpretation (physical or due to the gauge), the divergence of the characteristic speeds is troublesome in practice. The Courant-Friedrichs-Lewy stability condition implies that the time step $\Delta t$ of a numerical evolution should be $\Delta t<\Delta r / v$, with $\Delta r$ the spatial resolution and $v$ the maximum characteristic speed. Clearly, $\Delta t \rightarrow 0$ as $v \rightarrow \infty$, i.e., simulations must grind to a halt when the characteristic speeds diverge.

Therefore, our results suggest that the field equations of $k$-essence cannot be evolved starting from $k$-mouflage solutions. A possible practical solution to evolve the dynamics of $k$-mouflage may consist of using implicit methods $[49,50]$. However, the latter may not recover the system's true dynamics, as they might miss the smalltimescale features of the solution (and their cumulative secular effect, if any). In other words, implicit methods integrate out the UV details of the solution, which might be crucial for achieving a well-posed Cauchy evolution.

Conclusions.-We have shown that kinetic screening ( $k$-mouflage) of scalar effects occurs in isolated stars in $k$-essence, even when the stars are highly compact or relativistic, and the physically important requirement of regularity at the star's center is accounted for. $k$-mouflage solutions are stable to small perturbations, and also to large ones as long as they do not cause gravitational collapse. 
However, when large perturbations with the right sign for triggering collapse are applied to $k$-mouflage solutions, the evolution leads to diverging characteristic speeds for the scalar well before the formation of apparent black-hole or sound horizons. This divergence might not be pathological in itself, but prevents dynamical evolutions of the collapse of $k$-mouflage stars. Thus, $k$-essence loses predictability on $k$-mouflage configurations subject to these large perturbations. This is a serious flaw, as the theory cannot make predictions about the general time-dependent evolution of stars (including their collapse to a black hole), at least in $1+1$ dimensions. This is markedly different than in GR [51,52] or FJBD [32], where spherical dynamical simulations of compact objects present no such problems. Therefore, if kinetic screening exists, $k$-essence is (at best) incomplete in general dynamical settings. A UV completion of $k$-essence may render the time evolution of screened stars well posed. However, it is not guaranteed that $k$ mouflage solutions will still be present in such UV completions, see, e.g., [53]. Moreover, positivity bounds suggest that locality and/or Lorentz invariance may have to be violated to UV complete the theory [34], if screening solutions are to be present. However, one may attempt to modify the theory's equations in a UV-agnostic way inspired by dissipative hydrodynamics, possibly allowing for successfully evolving the dynamics [54].

L.t.H., M.B., M.C., and E.B. acknowledge support from the EU's H2020 ERC Consolidator Grant "GRavity from Astrophysical to Microscopic Scales" (Grant No. GRAMS-815673). C. P. acknowledges support from the Spanish Ministry of Economy and Competitiveness Grants No. AYA2016-80289-P and No. PID2019110301 GB-I00 (AEI/FEDER, UE). We thank J.M. Ibáñez, L. Lehner, and G. I. Montecinos for enlightening conversations on the well-posedness of the Cauchy problem.

[1] S. Weinberg, Rev. Mod. Phys. 61, 1 (1989).

[2] T. Clifton, P. G. Ferreira, A. Padilla, and C. Skordis, Phys. Rep. 513, 1 (2012).

[3] J. Ben Achour, M. Crisostomi, K. Koyama, D. Langlois, K. Noui, and G. Tasinato, J. High Energy Phys. 12 (2016) 100.

[4] M. Fierz, Helv. Phys. Acta 29, 128 (1956).

[5] P. Jordan, Z. Phys. 157, 112 (1959).

[6] C. Brans and R. H. Dicke, Phys. Rev. 124, 925 (1961).

[7] T. Kobayashi, M. Yamaguchi, and J. Yokoyama, Prog. Theor. Phys. 126, 511 (2011).

[8] G. W. Horndeski, Int. J. Theor. Phys. 10, 363 (1974).

[9] J. Gleyzes, D. Langlois, F. Piazza, and F. Vernizzi, Phys. Rev. Lett. 114, 211101 (2015).

[10] M. Crisostomi and K. Koyama, Phys. Rev. D 97, 084004 (2018).

[11] E. Berti et al., Classical Quantum Gravity 32, 243001 (2015).

[12] T. Damour and J. H. Taylor, Phys. Rev. D 45, 1840 (1992).
[13] C. M. Will, Theory and Experiment in Gravitational Physics (Cambridge University Press, Cambridge, 1993).

[14] C. M. Will, Living Rev. Relativity 17, 4 (2014).

[15] B. Abbott et al. (LIGO Scientific, Virgo Collaborations), Phys. Rev. D 100, 104036 (2019).

[16] A. Vainshtein, Phys. Lett. 39B, 393 (1972).

[17] E. Babichev and C. Deffayet, Classical Quantum Gravity 30, 184001 (2013).

[18] E. Babichev, C. Deffayet, and R. Ziour, Int. J. Mod. Phys. D 18, 2147 (2009).

[19] J. Khoury and A. Weltman, Phys. Rev. D 69, 044026 (2004).

[20] K. Hinterbichler and J. Khoury, Phys. Rev. Lett. 104, 231301 (2010).

[21] E. Belgacem et al., J. Cosmol. Astropart. Phys. 07 (2019) 024.

[22] T. Chiba, T. Okabe, and M. Yamaguchi, Phys. Rev. D 62 , 023511 (2000).

[23] C. Armendariz-Picon, V. F. Mukhanov, and P. J. Steinhardt, Phys. Rev. Lett. 85, 4438 (2000).

[24] B. Abbott et al. (LIGO Scientific, Virgo, Fermi-GBM, INTEGRAL Collaborations), Astrophys. J. Lett. 848, L13 (2017).

[25] B. Abbott et al. (LIGO Scientific, Virgo Collaborations), Phys. Rev. Lett. 119, 161101 (2017).

[26] P. Creminelli, M. Lewandowski, G. Tambalo, and F. Vernizzi, J. Cosmol. Astropart. Phys. 12 (2018) 025.

[27] P. Creminelli, G. Tambalo, F. Vernizzi, and V. Yingcharoenrat, J. Cosmol. Astropart. Phys. 05 (2020) 002.

[28] E. Babichev, J. High Energy Phys. 07 (2020) 038.

[29] M. Bezares, M. Crisostomi, C. Palenzuela, and E. Barausse, arXiv:2008.07546.

[30] While the Einstein frame is convenient for solving the equations numerically (see, e.g., [32,33]), when presenting results (unless stated otherwise), we transform back to the Jordan frame, where the physical interpretation is clearer; e.g., we plot results vs the Jordan-frame areal radius $r$, which differs by a conformal factor from the Einstein-frame radius $\tilde{r}$.

[31] Without gravity, this choice leads to superluminal propagation and nonanalytic $2 \rightarrow 2$ scattering amplitudes in the forward limit [34], but see [35] for recent developments.

[32] E. Barausse, C. Palenzuela, M. Ponce, and L. Lehner, Phys. Rev. D 87, 081506(R) (2013).

[33] C. Palenzuela, E. Barausse, M. Ponce, and L. Lehner, Phys. Rev. D 89, 044024 (2014).

[34] A. Adams, N. Arkani-Hamed, S. Dubovsky, A. Nicolis, and R. Rattazzi, J. High Energy Phys. 10 (2006) 014.

[35] L. Alberte, C. de Rham, S. Jaitly, and A. J. Tolley, Phys. Rev. D 102, 125023 (2020).

[36] E. Babichev, V. Mukhanov, and A. Vikman, J. High Energy Phys. 02 (2008) 101.

[37] P. Brax and P. Valageas, Phys. Rev. D 90, 123521 (2014).

[38] This nonlinear regime may seem problematic from an EFT viewpoint. However, [39] (without gravity) and [40] (with gravity) showed that quantum corrections are under control in the nonlinear regime.

[39] C. de Rham and R. H. Ribeiro, J. Cosmol. Astropart. Phys. 11 (2014) 016.

[40] P. Brax and P. Valageas, Phys. Rev. D 94, 043529 (2016). 
[41] E. Babichev, C. Deffayet, and R. Ziour, Phys. Rev. D 82, 104008 (2010).

[42] E. Babichev and M. Crisostomi, Phys. Rev. D 88, 084002 (2013).

[43] S. Valdez-Alvarado, C. Palenzuela, D. Alic, and L. A. Ureña-López, Phys. Rev. D 87, 084040 (2013).

[44] This is not trivial. Even for these initial data, numerical evolutions break with standard finite-difference or even soft shock-capturing methods, presumably as a result of strong microshocks in the scalar field [45-47] which form even from smooth initial data. This suggests that Eqs. (2) and (3) only allow for weak solutions (i.e., solutions to the integral version of the system), which we successfully obtain by using HRSC methods.

[45] H. S. Reall, N. Tanahashi, and B. Way, Phys. Rev. D 91, 044013 (2015).
[46] E. Babichev, J. High Energy Phys. 04 (2016) 129.

[47] L. Bernard, L. Lehner, and R. Luna, Phys. Rev. D 100, 024011 (2019).

[48] P. Figueras and T. França, Classical Quantum Gravity 37, 225009 (2020).

[49] L. Pareschi and G. Russo, J. Sci. Comput. 25, 129 (2005).

[50] C. Palenzuela, L. Lehner, O. Reula, and L. Rezzolla, Mon. Not. R. Astron. Soc. 394, 1727 (2009).

[51] M. Shibata and K. Uryū, Phys. Rev. D 61, 064001 (2000).

[52] F. Pretorius, Phys. Rev. Lett. 95, 121101 (2005).

[53] C. Burrage, B. Coltman, A. Padilla, D. Saadeh, and T. Wilson, arXiv:2008.01456.

[54] G. Allwright and L. Lehner, Classical Quantum Gravity 36, 084001 (2019). 